\title{
Viral misinformation threatens public health
}

\author{
Cite as: CMAJ 2017 December 18;189:E1567. doi: 10.1503/cmaj.109-5536
}

Posted on cmajnews.com on Nov. 30, 2017.

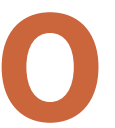

utbreaks of fake news and distorted evidence are threatening the biggest public health achievements of the past century, warns Dr. Brittany Seymour, an assistant professor of oral health policy and epidemiology at Harvard University. But mapping these "digital pandemics" is giving researchers a better idea of how to set the record straight.

A mind-blowing amount of information is shared on the internet, Seymour explained at a recent Ontario Public Health Association forum. Faced with information overload, "objective facts are less influential in shaping public opinion than appeals to emotion and personal belief."

With $80 \%$ of people seeking health information online, viral misinformation poses a growing threat to public health, she said. "One of the best examples is vaccines." In 2014, the United States had one of the largest measles outbreaks in a generation. "We've been able to trace that, in part, to parents who found scary information on the Internet and opted not to vaccinate their children," Seymour said. Despite researchers and institutions issuing corrective information, "counter efforts have not been as effective as we'd like." Three years on, "we see the Centers for Disease Control responding to a whole list of concerns related to the safety and effectiveness of childhood vaccination," she said.

Meanwhile, there is growing controversy around water fluoridation, spurred by a 2014 study with "severe limitations" that linked the chemical to lower IQs in children. "Within a matter of hours from its release, the article created a flood of posts on Twitter, and was covered in pop media news stories across the Internet, generating tens of thousands of views and shares," Seymour said. Her research mapping these online conversations reveals that "similar to the anti-vaccination community, a small but vocal and very tightly knit network is likely driving the anti-fluoridation lobby."

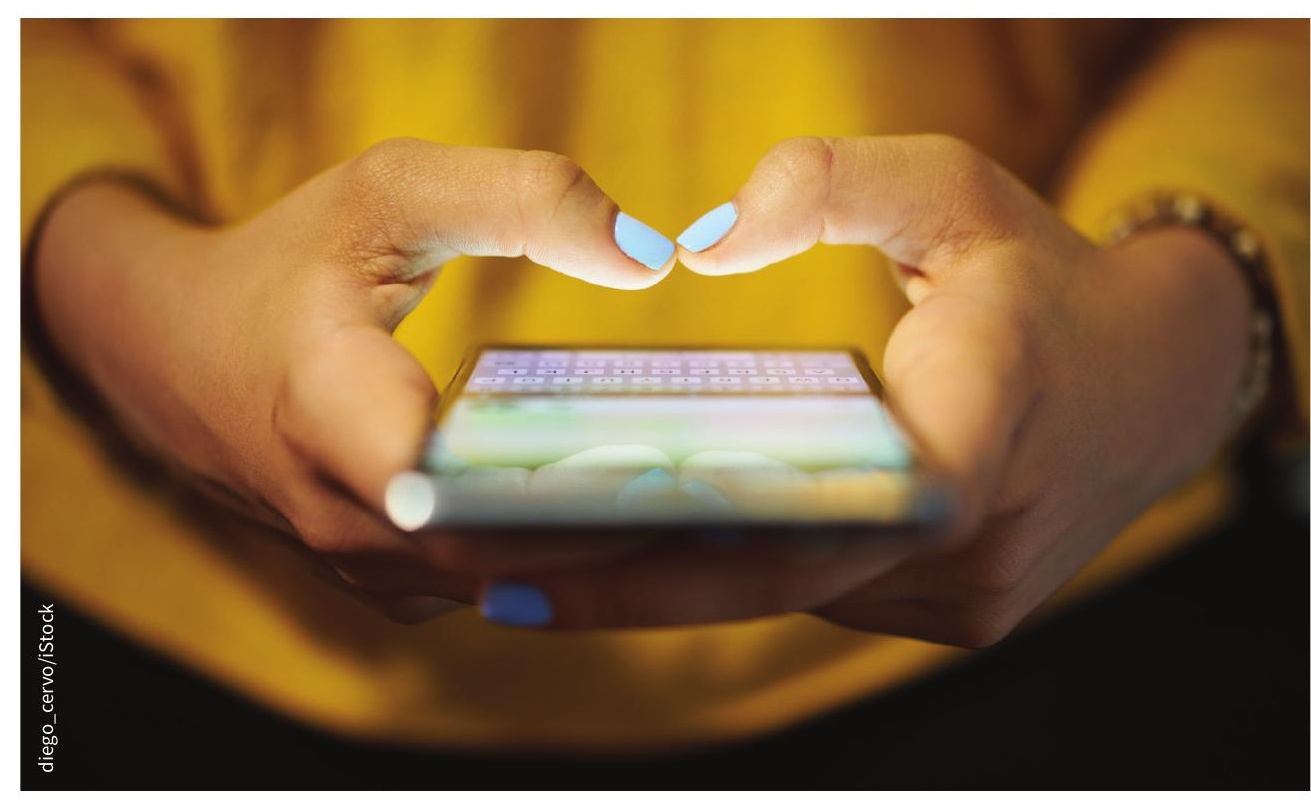

Information overload is driving people to trust social influence over evidence on health matters.

Highly connected networks have strict sets of social norms and quickly reject any dissenter or contradictory piece of evidence, Seymour explained. Members frequently share and cite studies to back their arguments, but often the studies were not linked to or were several clicks away, and "the risk of evidence becoming misrepresented increases with each link that takes the reader further from the original article," she said.

Curiously, the public health community clusters together in a similar way, Seymour added. "We really like each other and link to each other a lot, but we're not getting engagement beyond our own echo chamber."

Targeting social influencers - the members of networks with the most ties to different social clusters - may help cross that divide. This is already happening organically, she noted, citing the example of a Connecticut dentist promoting a "back to school" checklist that included a dental check-up. "Rather than tweeting it out to her own followers on Twitter, she tweeted at a very influential mom group, and the mom in charge retweeted the checklist," Seymour said. "That's the way we need to start thinking about how we communicate and who we target."

It's similar to the approach the Harvard School of Public Health used in the 1980s to reduce drunk driving by getting television shows like Cheers to popularize the concept of a "designated driver," she added. "They didn't show statistics; they created a social norm, and not only did we see a drastic reduction in alcohol-related accidents and deaths, but 'designated driver' became a household term."

As the balance of influence has shifted from broadcasters to individuals, public health must also shift "from how can we get evidence-based information to our target audience, to how we can get our audience to share our evidence-based information."

Lauren Vogel, CMAJ 